\title{
Result of 10 Years of Experience in Echo-Guided Kidney Biopsy Punctures in the Nephrology Department of the Martigues Hospital Center, France
}

\author{
Aboubacar Sidiki Fofana1*, Magara Samaké1, Seydou Sy ${ }^{2,3}$, Sah Dit Baba Coulibaly4, \\ Christophe Bouaka ${ }^{5}$, Ghassan Alchahin ${ }^{5}$, Delphine Haussaires, Simona Dorina Boncila5, \\ Hamadoun Yattara ${ }^{2,3}$, Moctar Coulibaly6, Atabième Kodio ${ }^{2}$, Modi Sidibe'2, Saharé Fongoro's,3 \\ ${ }^{1}$ Nephrology Unit of the Fousseyni DAOU Hospital, Kayes, Mali \\ ${ }^{2}$ Nephrology and Hemodialysis Department of the University Teaching Hospital of Point-G, Bamako, Mali \\ ${ }^{3}$ Faculty of Medicine and Odontostomatology of the University of Sciences, Techniques and Technologies of Bamako, Bamako, \\ Mali \\ ${ }^{4}$ Nephrology Unit of Somino DOLO Hospital, Mopti, Mali \\ ${ }^{5}$ Nephrology and Hemodialysis Department of the Martigues Hospital, Martigues, France \\ ${ }^{6}$ Nephrology Unit of Mali Gavardo Hospital, Sébénikoro, Bamako, Mali \\ Email: ^fofaboubacarsidiki@gmail.com
}

How to cite this paper: Fofana, A.S., Samaké, M., Sy, S., Coulibaly, S.D.B., Bouaka, C., Alchahin, G., Haussaire, D., Boncila, S.D., Yattara, H., Coulibaly, M., Kodio, A., Sidibe, M. and Fongoro, S. (2021) Result of 10 Years of Experience in Echo-Guided Kidney Biopsy Punctures in the Nephrology Department of the Martigues Hospital Center, France. Open Journal of Nephrology, 11, 144-155.

https://doi.org/10.4236/ojneph.2021.112012

Received: March 12, 2021

Accepted: April 23, 2021

Published: April 26, 2021

Copyright () 2021 by author(s) and Scientific Research Publishing Inc. This work is licensed under the Creative Commons Attribution International License (CC BY 4.0).

http://creativecommons.org/licenses/by/4.0/

\section{Abstract}

Introduction: Percutaneous renal biopsy (PRB) is an essential procedure for the diagnosis and therapeutic management of many primary or secondary nephropathies. Objectives: To identify the indications, to determine the profile of the diagnosed nephropathies and to evaluate the short-term complications related to the practice of echo-guided PRB at the Martigues hospital center. Methodology: This was a retrospective and descriptive study carried out on the records of patients who underwent echo-guided native kidney biopsy from January 1, 2010 to December 31, 2019 in the nephrology department of the Martigues Hospital. Results: The analysis of 123 cases of echoguided PRB involved 76 men and 47 women with a sex ratio of 1.6. The mean age was $55.92 \pm 17.80$ with age extremes of 16 and 87 years. Glomerular syndromes were the main indication with 42 cases of nephrotic syndrome $(34.1 \%)$, 15 cases of nephritic syndrome (12.2\%), 11 cases of rapidly progressive glomerulonephritis syndrome (8.9\%), and 6 cases of recurrent macroscopic hematuria syndrome $(4.9 \%)$. The histological findings were 47 cases of primary glomerular lesions (38.3\%), 32 cases of nephroangiosclerosis lesions (26\%), 24 cases of secondary glomerulopathy (19.5\%), 9 cases of interstitial nephritis 
(7.3\%), 2 cases of myelomatous nephropathy (1.6\%), and 9 cases $(7.3 \%)$ of unclassified histological lesions. Twenty-two hypertensive patients (40.7\%) had nephroangiosclerosis lesions $(\mathrm{p}=0.001)$. The follow-up was simple in 119 patients (96.7\%). Macroscopic hematuria was noted in 4 patients $(3.3 \%)$. It was associated with a perirenal hematoma in 2 patients including 1 transfused case. Conclusion: Our data provide an important contribution to the understanding of the prevalence and clinical presentation of renal diseases in the nephrology department of the Martigues hospital center.

\section{Keywords}

Renal Puncture Biopsy, Nephrology, Martigues Hospital Center

\section{Introduction}

Percutaneous renal biopsy (PRB) is an essential procedure for the diagnosis and therapeutic management of many primary or secondary nephropathies. It is a very important examination in nephrological practice to establish the precise diagnosis of nephropathy, to provide prognostic indications and to guide the treatment [1]. According to the results of a multicenter survey carried out in 2009 in metropolitan France, native kidney biopsy was performed in 73 departments with an annual number of procedures ranging from several hundred to less than ten [2]. This practice of PRB in nephrology is not performed in many countries with limited resources and particularly in Africa because of the lack of technical facilities [3].

Described for the first time in 1951 by Iversen and Brun [4], the renal biopsy technique has undergone many changes over time. Improvements in the last decades through real-time ultrasound guidance and the use of the automatic gun have transformed renal biopsy into a safe and informative technique [5]. Some recent studies report a success rate (diagnostic biopsies) of more than $99 \%$ and less than $0.1 \%$ of life-threatening complications [2]. PRB is not a trivial procedure, but it remains invasive with various complications, mainly bleeding [6].

The objectives of this study were to identify the indications, to determine the profile of diagnosed nephropathies and to evaluate the complications related to the practice of echo-guided PRB in the nephrology department of the Martigues hospital center.

\section{Patients and Methods}

\subsection{Type and Period of Study}

This was a retrospective and descriptive study of PRB data dating from January 1, 2010 to December 31, 2019 inclusive, i.e., a 10-year period.

It was conducted in the nephrology and hemodialysis department of the Martigues Hospital Center located in the Rayette, France, in the department of Rhone mouth, Provence-Alpes-Côte d'Azur region. 


\subsection{Eligibility Criteria}

We included all patient records that underwent echoguided native kidney biopsy during the study period. They were selected from the pathology registry of PRB results of the nephrology department.

The records of transplanted patients who underwent renal graft biopsy and cases of PRB performed under CT scan and transjugular route were not included in this study.

\subsection{Renal Biopsy Sampling Technique}

The sampling technique used was percutaneous renal biopsy under ultrasound guidance. This is the most widely used and oldest method of renal biopsy. It has the advantage of simplicity and low cost due to a short hospital stay of 24 to 48 hours. The ultrasound was initially performed by the department's physicians before the day of the procedure. Antiplatelet drugs were stopped, aspirin 7 days, clopidogrel 10 days before the procedure. In case of anticoagulant treatment, a relay was made with heparin.

The patient was placed prone on a hard surface and on a rolled pillow serving as a log compressing the abdomen and fixing the kidney. The puncture site is preferably chosen at the lower pole of the left kidney, which is less mobile than the right kidney, and with a low risk of injury to the inferior vena cava.

We used Bard Monopty single-use automatic guns with 16-gauge needles. After local anesthesia with xylocaine of the different cutaneous-musculoaponeurotic planes on the puncture path followed by a small local skin incision with a scalpel, the sampling was performed under real-time ultrasound tracking of the renal parenchymal fragments.

After the biopsy, the patient remained at rest in dorsal recumbency for 24 hours.

Monitoring included assessment of pain, diuresis, and hematuria, as well as regular measurement of blood pressure, heart rate, and use of urine strips.

On the paraclinical level, a control haemogram was requested between 12 and 24 hours after the procedure. In case of macroscopic hematuria more or less associated with pain or deglobulization on the blood count, a renal ultrasound or even a renal CT scan was performed to look for a significant perirenal hematoma.

Patients were instructed to limit physical activity for 10 to 15 days after discharge. Similarly, it was recommended not to resume antiaggregant or anticoagulant treatments in the 7 to 10 days following the procedure.

\subsection{Sample Collection Procedures}

Two (02) fragments were usually collected. The first was quickly placed in $2.5 \%$ glutaraldehyde fluid for light microscopy and the second in Michel's fluid for immunofluorescence. The samples were sent to the University Teaching Hospital of Timone in Marseille through the laboratory of the Martigues center hospit- 
al for anatomopathological reading. The result is sent to us by e-mail and/or by mail within 3 to 7 days.

\subsection{Parameters Studied}

Data were collected from the PBR result register and the patients medical records. We noted the following elements: demographic aspects (age, sex), clinical evaluation (history, physical examination), and paraclinical evaluation (blood count in search of anemia and/or thrombocytopenia), coagulation workup (prothrombin time, active partial thromboplastin time, primary hemostasis study).

The platelet occlusion time was systematically requested in all patients and its normality was one of the sine qua non conditions for the performance of echoguided PRB.

In terms of indication for PRB; the main syndromes were defined as follows [1]:

- Nephrotic syndrome (NS): proteinuria greater than $3 \mathrm{~g} / \mathrm{d}$ and hypo-albuminemia less than $30 \mathrm{~g} / \mathrm{l}$; apart from typical NS in children aged 1 - 10 years, and diabetic retinopathy on fundus without hematuria.

- Acute nephritic syndrome: abrupt onset of acute renal failure (ARF) of variable intensity, hypertension and proteinuria sometimes of nephrotic volume.

- Rapidly progressive glomerulonephritis (RPGN) syndrome: rapidly worsening AKI associated with an inaugural macroscopic hematuria, the usual absence of hypertension, and proteinuria.

- Permanent non-nephrotic proteinuria isolated or associated with microscopic hematuria.

- Tubulointerstitial nephropathy syndrome (TNIS): proteinuria usually $<1 \mathrm{~g} /$ day without hypertension or edema, more or less associated with hematuria and AKI.

- Vascular Nephropathy Syndrome (VNS): hypertension in the foreground, absence of major abnormality on urine sediment examination, and the presence of often severe and rapidly progressive renal failure.

- Isolated acute renal failure (ARF): recent elevation of serum creatinine, without evidence of chronicity (absence of anemia and hypocalcemia and kidneys maintaining normal size and good differentiation on renal ultrasound).

- Systemic diseases associated with proteinuria or renal failure: multiple systemic diseases such as lupus, vasculitis, amyloidosis, myeloma, sarcoidosis or drug-related complications can be diagnosed by renal biopsy [7].

- Unexplained chronic renal failure: presence of criteria for chronic renal disease with normal sized and differentiated kidneys. Renal biopsy can provide important information, especially if renal failure is accelerating. In this case, it may reveal lesions that justify specific treatment (extracapillary proliferation, cholesterol emboli, interstitial nephropathy) [1].

The complications sought were transient macroscopic hematuria, uncomplicated peri-renal hematomas, hemorrhages requiring transfusion or even surgical 
hemostasis or nephrectomy [8].

\subsection{Data Entry and Analysis}

Data entry was done on the EPI-DATA 3.1 software.

Data were analyzed on SPSS 21.0 software.

The statistical test of comparison was Pearson's chi-square with Yates correction. A p value less than or equal to 0.05 was considered significant.

\subsection{Ethical Consideration}

The patient was informed of the benefit and risks of the procedure, followed by a signature on the informed consent form before the PBR was performed.

The anonymity of each participant's survey form was strictly respected.

\section{Results}

During the period from January 1, 2010 to December 31, 2019 inclusive, the Nephrology Department of the Martigues hospital center realized a total of 142 biopsy punctures on native kidney.

Nineteen (19) renal biopsy punctures were excluded from this study, which concerned cases of transjugular biopsy (9), renal biopsy under scanner (3) and renal biopsy whose result was not contributory (7).

One hundred and twenty-three (123) ultrasound-guided renal biopsy results were analyzed.

In terms of annual realization, there was an increase in frequency during the last 3 years, i.e. 16 cases in 2017 (13\%), 18 cases (14.6\%) in 2018 and 2019 respectively (Figure 1).

The male sex was in the majority (76 males versus 47 females), i.e. $61.8 \%$ with a sex-ratio of 1.6.

The mean age was 55.92 years \pm 17.80 with age extremes of 16 and 87 years. The age range 25 - 64 years represented 66 patients, i.e. 53.7\% of the cases (Table 1).

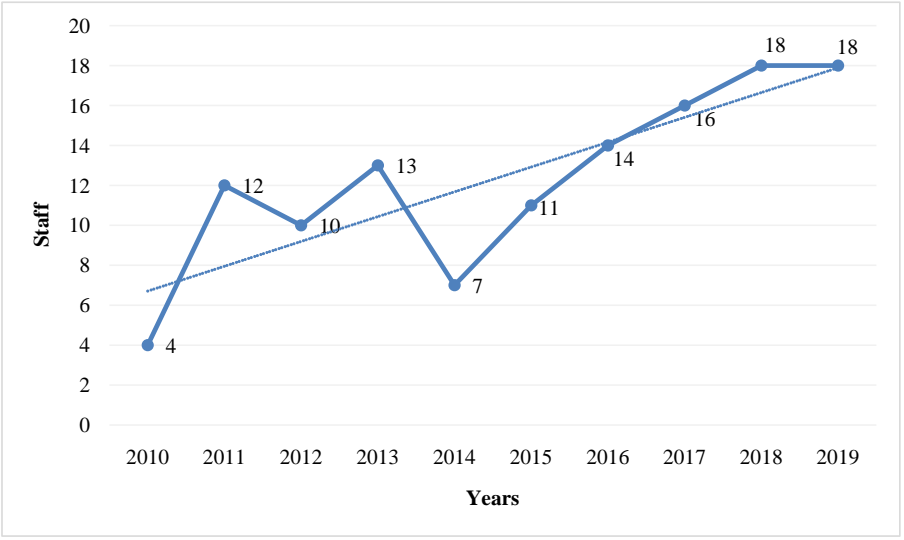

Figure 1. Annual number of ultrasound-guided renal biopsies. 
Table 1. Distribution of patients by age.

\begin{tabular}{ccc}
\hline Age range in years & Staff & Percentage \\
\hline $15-24$ & 11 & 8.9 \\
$25-64$ & 66 & 53.7 \\
65 years and older & 46 & 37.4 \\
Total & 123 & 100 \\
\hline
\end{tabular}

A history of hypertension and diabetes was found in 54 patients (43.9\%) and 27 patients (22\%) respectively.

Regarding the indications for PRB, glomerular syndromes were the main reason with 42 cases of nephrotic syndrome (34.1\%), 15 cases of the nephritic syndrome (12.2\%), 11 cases of rapidly progressive glomerulonephritis syndrome (8.9\%), and 6 cases of recurrent macroscopic hematuria syndrome (4.9\%) (Figure 2).

The NS was impure in 31 patients (3.8\%). The mean 24-hour proteinuria in patients with glomerular syndrome was $2.767 \pm 1.496 \mathrm{~g} / 24$ hours.

Other reasons for PRB use were 12 cases of vascular nephropathy syndrome (9.8\%), 11 cases of unexplained CKD (8.9\%), 7 cases of systemic diseases associated with proteinuria or renal failure (5.7\%), and 7 cases of isolated AKI (5.7\%).

The anatomopathological result was made possible through the examination of 02 fragments of which 1 for light microscopy and a second for immunofluorescence. The average number of glomeruli in light microscopy was $13.097 \pm$ 8.585 with an average of glomeruli in "sealing bread" at $2.336 \pm 2.465$. On immunofluorescence, there was an average of $6252 \pm 4827$ glomerules.

The analysis of the histological results showed 47 cases of primary glomerular lesions (38.3\%), 32 cases of nephroangiosclerosis lesions (26\%), 24 cases of secondary glomerulopathies (19.5\%), 9 cases of interstitial nephritis (7.3\%), 2 cases of myelomatous nephropathy (1.6\%) and 9 cases (7.3\%) of unclassified histological lesions (Table 2).

The primary glomerular lesions were IgA-deposited mesangial nephropathy (14), extracapillary glomerulonephritis (8), extramembranous glomerulonephritis (12), minimal glomerular lesions (8), membranoproliferative glomerulonephritis (3), and segmental and focal hyalinosis (2). Glomerulopathies secondary to general diseases were mainly diabetic nephropathy (9), followed by renal amyloidosis (7), ANCA vasculitis (4), systemic lupus erythematosus (3) and 1 case of rheumatoid purpura nephropathy. Twenty-two hypertensive patients had the nephroangiosclerosis lesions in $40.7 \%$ of cases ( $p=0.001$, Table 3 ).

The unclassified histological lesions were fibrosis (4), extensive mesangliolysis (1), glomerular hypertrophy (1), C3-deposit glomerulopathy (1), acute post-streptococcal glomerulonephritis (1) and light chain disease (1).

In terms of short-term evolution after the procedure, the after-effects were simple in 119 patients (96.7\% of cases). Macroscopic hematuria was noted in 4 patients (3.3\%). It was associated with a perirenal hematoma in 2 patients, including 1 transfused patient. 


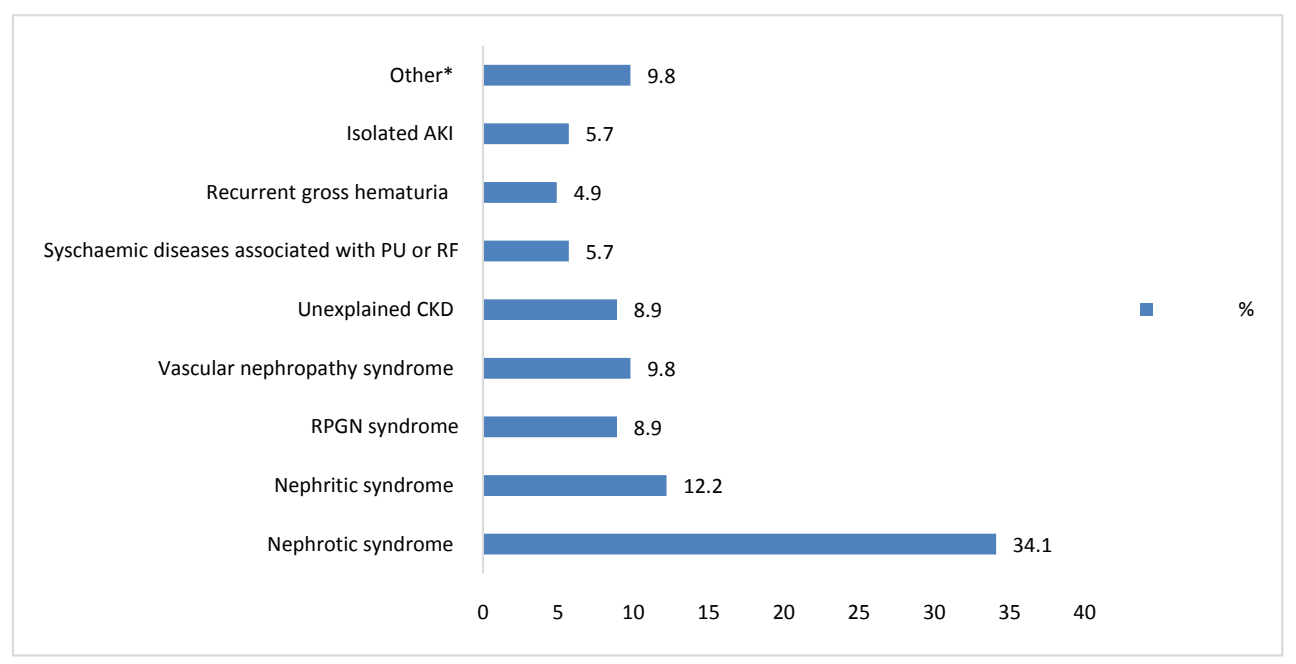

Figure 2. Clinical indications for percutaneous renal biopsy. PU: proteinuria; RF: renal failure; RPGN: rapidly progressive glomerulonephritis; AKI: acute kidney injury; CKD: chronique kidney disease; ${ }^{\star}$ Other: persistent hematuria in a diabetic setting, AKI associated with isolated proteinuria, and tubular-interstitial nephropathy syndrome.

Table 2. Renal pathologies.

\begin{tabular}{|c|c|c|}
\hline Renal pathologies & Staff & Percentage \\
\hline \multicolumn{3}{|l|}{ Primary glomerular lesions } \\
\hline - IgA nephropathy & 14 & 11.4 \\
\hline - EMGN & 12 & 9.8 \\
\hline - MGL & 8 & 6.5 \\
\hline - ECGN & 8 & 6.5 \\
\hline - MPGN & 3 & 2.4 \\
\hline$-\mathrm{SFH}$ & 2 & 1.6 \\
\hline \multicolumn{3}{|l|}{ Secondary glomerular lesions } \\
\hline - Diabetic nephropathy & 9 & 7.3 \\
\hline - Renal amyloidosis & 7 & 5.7 \\
\hline - ANCA vasculitis & 4 & 3.3 \\
\hline - Lupus nephropathy & 3 & 2.4 \\
\hline - Kidney disease in RP & 1 & 0.8 \\
\hline Nephroangiosclerosis & 32 & 26.0 \\
\hline Interstitial nephritis & 9 & 7.3 \\
\hline Myeloma nephropathy & 2 & 1.6 \\
\hline *unclassified & 9 & 7.3 \\
\hline Total & 123 & 100.0 \\
\hline
\end{tabular}

EMGN: Extramembranous glomerulonephritis; MGL: Minimal glomerular lesions; ECGN: Extracapillary glomerulonephritis; MPGN: Membranous proliferative glomerulonephritis; SFH: Segmental and focal hyalinosis; RP: Rheumatoid purpura; * unclassified: fibrosis (4), extensive mesangliolysis (1), glomerular hypertrophy (1), C3 deposition glomerulopathy (1), acute post streptococcal glomerulonephritis (1) and light chain disease (1). 
Table 3. Distribution of patients by history of hypertension and nephroangiosclerosis.

\begin{tabular}{llccc}
\hline & & \multicolumn{3}{c}{ Nephroangiosclerosis } \\
\cline { 3 - 5 } & & Yes & No & Total \\
\hline \multirow{2}{*}{ Hypertensive patients } & Yes & $22(40.7 \%)$ & $32(59.3 \%)$ & $54(100.0 \%)$ \\
& No & $10(14.5 \%)$ & $59(85.5 \%)$ & $69(100.0 \%)$ \\
\hline
\end{tabular}

chi-square $=10.843$; degree of liberty $=1 ; \mathrm{p}=0.001$.

Renal biopsy was the reason for hospitalization in 81 patients $(65.9 \%)$ with a mean duration of 2.79 days \pm 1.31 . It was performed in 42 patients $(34.1 \%)$ of the cases during traditional hospitalization with an average length of hospitalization of 12.67 days \pm 7.19 .

\section{Discussion}

The nephrology department of the Martigues hospital center started PRB for the first time in 2007. This practice of renal biopsy in nephrology is not performed in many developing countries and particularly in Africa because of the insufficiency of the technical platform [3].

This study shows an increase in the number of PRB during the last 4 years, in parallel with the increase of the department's activities.

The average age of the patients was 55.92 years \pm 17.80 , with extremes of 16 and 87 years.

A series by Yong on the analysis of renal biopsy data from a single center in the rural Midlands region of China from 1996 to 2010 found a mean age of 33.1 years (extreme 16 - 72 years) [9]. In contrast, in Morocco in 2016, the mean age of patients was $39 \pm 17$ years, with extremes of 3 and 82 years [10].

Nowadays, we are witnessing an aging of the French population during this last decade with a median age in 2020 at 41.1 versus 38.8 in 2010 according to data from the National Institute of Statistics and Economic Studies [11].

After analysis of clinical, biological and histological data, we confirmed the definitive anatomical and clinical diagnosis in our patients.

From the clinical presentation of glomerular damage, nephrotic syndrome was the first indication for PBR with a frequency of $34.1 \%$. According to the literature many other authors found a predominance of nephrotic syndrome with a higher frequency than ours, as the case of these studies performed in South Africa [3] and Brazil [12] with respectively 52.5\% and $39 \%$ of cases.

In terms of histological findings, nephroangisoclerosis lesions were predominant, i.e. 26\% (32 cases/123). This was a case of malignant nephroangisoclerosis in a young 43-year-old patient with irregular follow-up, who presented clinically with malignant hypertensive encephalopathy, rapidly progressive renal failure associated with nephrotic proteinuria.

The malignant nephroangisoclerosis is an anatomical-clinical syndrome that can complicate essential hypertension (neglected, discontinued) in $2 / 3$ of cases and secondary hypertension in 1/3 of cases [13]. 
IgA-deposited mesangial nephropathy was the most common histological entity of primary glomerulopathy, representing $11.4 \%$ (14 cases). The clinical presentation in this case was in order of frequency of nephrotic syndrome, recurrent macroscopic hematuria, nephritic syndrome and isolated proteinuria.

Houda in Morocco found 3.2\% of cases of IgA nephropathy versus $34.1 \%$ in the Young study in China [9] [10].

This nephropathy is the world's leading cause of primary glomerulonephritis. Its incidence varies significantly with maximum values in Asia (5.7 new cases per 100,000 inhabitants) and minimum values in Africa (0.2/100,000 inhabitants). In France, the incidence is intermediate $(2.5 / 100,000)$ [14].

Clinically, the main symptom in IgA nephropathy is hematuria ( $90 \%$ of patients). Hematuria is typically macroscopic in the form of flare-ups during episodes of respiratory mucosal infections. But the most common form is chronic microscopic hematuria. The presence of proteinuria greater than $1 \mathrm{~g} / 24$ hours is less frequent. High proteinuria ( $>3 \mathrm{~g} / 24$ hours) more or less nephrotic may be present [15].

We noted 9 cases of diabetic nephropathy in all the samples tested.

Among the 27 type II diabetic patients without retinopathy with a GFR less than $60 \mathrm{ml} / \mathrm{min}$, with significant proteinuria, more or less associated with hematuria, the prevalence of diabetic nephropathy was $33.3 \%$. Other histological lesions in these diabetic patients were nephroangisclerosis (12), interstitial nephritis (2), renal amyloidosis (2), IgA-deposited nephropathy (1) and membranoproliferative glomerulonephritis (1).

According to the results of the ENTRED study from 2001 to 2007, the prevalence of diabetic CKD in a population of patients with type 2 diabetes in France is estimated at $29 \%$ [16].

Hematuria, a sign of glomerular inflammation, is not the rule in diabetic nephropathy. It should be checked for persistence and the absence of associated bacteriuria. Its presence should lead to a search for other arguments for glomerulopathy other than diabetic [17].

ANCA vasculitis with pauci-immune extracapillary glomerulonephritis represented 3.3\%, i.e. 3 cases of MPO ANCA versus 1 case of PR3 ANCA. The clinical presentation was in all cases a rapidly progressive glomerulonephritis syndrome. The BVAS score was not systematically evaluated.

Seven cases (5.7\%) of amyloidosis were noted in this study in subjects older than 60 years (Table 4). They were of type AL in 6 patients versus 1 case of AA amyloidosis.

Renal involvement is frequent in multisystemic amyloidosis, which accounts for about $3 \%$ of all nephropathies. It is thus observed in $90 \%$ of AA amyloidosis (derived from a serum precursor called SAA for serum amyloid associated protein precursor) and in nearly 50\% of AL amyloidosis cases (derived from immunoglobulin light chains). Its incidence can reach $15 \%$ in renal biopsies performed in subjects over 60 years of age because of the high frequency of AL amyloidosis at this age [18]. 
Table 4. Distribution of patients by renal pathology and age group.

\begin{tabular}{ccccc}
\hline \multirow{2}{*}{ Renal pathologies } & \multicolumn{3}{c}{ Age range in years } & Total \\
\cline { 2 - 4 } & $15-24$ & $25-64$ & 65 and over & \\
\hline Nephoangiosclerosis & - & $17(53.1 \%)$ & $15(46.9 \%$ & $32(100 \%)$ \\
EMGN & $2(16.7 \%)$ & $6(50 \%)$ & $4(33.3 \%)$ & $12(100 \%)$ \\
ECGN & $1(12.5 \%)$ & $1(12.5 \%)$ & $6(75 \%)$ & $8(100 \%)$ \\
MGL & $2(25 \%)$ & $4(50 \%)$ & $2(25 \%)$ & $8(100 \%)$ \\
IgA nephropathy & $1(7.1 \%)$ & $9(64.3 \%)$ & $4(28.6 \%)$ & $14(100 \%)$ \\
SFH & - & $2(100 \%)$ & - & $2(100 \%)$ \\
Diabetic nephropathy & $1(11.1 \%)$ & $4(44.4 \%)$ & $4(44.4 \%)$ & $9(100 \%)$ \\
Amyloidosis & - & $6(85.7 \%)$ & $1(14.5 \%)$ & $7(100 \%)$ \\
Interstitial nephritis & - & $1(50 \%)$ & $1(50 \%)$ & $2(100 \%)$ \\
Lupus nephropathy & $1(33.3 \%)$ & $2(66.7 \%)$ & - & $3(100 \%)$ \\
Myeloma nephropathy & - & $1(50 \%)$ & $1(50 \%)$ & $2(100 \%)$ \\
ANCA vasculitis & $1(25 \%)$ & - & $3(75 \%)$ & $3(100 \%)$ \\
Kidney disease in RP & - & $1(100 \%)$ & - & $1(100 \%)$ \\
non classified & $1(11.1 \%)$ & $5(55.6 \%)$ & $3(33.3 \%)$ & $9(100 \%)$ \\
\hline
\end{tabular}

The aftermath of the renal biopsy was simple in the majority of patients (96.7\%). The procedure was complicated by macroscopic hematuria in 4 patients. It was associated with a perirenal hematoma on the CT scan in 2 patients, 1 of whom required a blood transfusion for a major deglobulization.

Gross hematuria is the most frequent complication of renal biopsy. Most often, it only warrants medical supervision and a prescription for copious fluids. Hematomas are usually asymptomatic and small. However, they may cause significant pain and require transfusion if they are large with deglobulization. Immediate ultrasound monitoring after renal biopsy is still controversial [19].

The cases of hemorrhagic complications in this study involved a 75-year-old man and 3 women aged 16, 54 and 65 years.

Several studies have identified factors associated with bleeding complications of percutaneous ultrasound-guided renal biopsies. Female gender and age (higher risk for the two extremes of age $<20$ years and $>70$ years), regardless of histologic diagnosis, were consistently found [20].

The limitations of this study are the small sample size (123 cases over 10 years), the monocentric nature, and the absence of electron microscopy.

\section{Conclusion}

The data from this study make an important contribution to the understanding of the prevalence and clinical presentation of renal disease at the Martigues hospital center. The histological lesions are dominated by nephroangiosclerosis and glomerulopathies with an important primary component. 


\section{Conflicts of Interest}

The authors declare no conflicts of interest regarding the publication of this paper.

\section{References}

[1] Kourilsky, O. (2014) La biopsie rénale. In: Kanfer, A., Kourilsky, O., Peraldi, M.-N. and Combe, C., Eds., Néphrologie et troubles hydroélectrolytiques, Elsevier Masson, Paris. https://doi.org/10.1016/B978-2-294-73759-6.00018-3

[2] Bollée, G., Moulin, B., Martinez, F., Meulders, Q., Rougier, J.P., Baumelou, A., et al. (2012) Pratique de la biopsie rénale: résultat d'une enquête en France, revue de la littérature et recommandations. Néphrologie \& Thérapeutique, 8, 168-176. https://doi.org/10.1016/j.nephro.2011.12.005

[3] Okpechi, I., Swanepoel, C. and Duffield, M. (2011) Patterns of Renal Disease in Cape Town South Africa: A 10-Year Review of a Single-Centre Renal Biopsy Database. Nephrology Dialysis Transplantation, 26, 1853-1861. https://doi.org/10.1093/ndt/gfq655

[4] Iversen, P. and Brun, C. (1951) Aspiration Biopsy of the Kidney. The American Journal of Medicine, 3, 324-330. https://doi.org/10.1016/0002-9343(51)90169-6

[5] Lefaucheur, C., Nochy, D. and Bariety, J. (2009) Biopsie rénale: Techniques de prélèvement, contre-indications, complications. Néphrologie \& Thérapeutique, 5, 331-339. https://doi.org/10.1016/j.nephro.2009.02.005

[6] Heng, A.E., Ackoundou-Nguessan, C., Gazuy, N., Deteix, P. and Souweine, B. (2005) Place de la ponction biopsie rénale dans l'insuffisance rénale aiguë en réanimation. Réan, 14, 483-490. https://doi.org/10.1016/j.reaurg.2005.09.009

[7] Weening, J.J. (2004) The Classification of Glomerulonephritis in Systemic Lupus Erythematosus Revisited. Journals of the American Society of Nephrology, 2, 241-250. https://doi.org/10.1097/01.ASN.0000108969.21691.5D

[8] Rabant, M., Snanoudj, R., Krug, P. and Martinez, F. (2017) Méthode et technique de la biopsie rénale. In: Traité de néphrologie, E Thervet, Paris, 734.

[9] Wang, Y.-T., Zhou, C.-Y., Zhu, T.-C., Yang, J., Zhang, Y., Xu, Q.-Y., et al. (2013) Analysis of Kidney Biopsy Data from a Single Center in the Midland Rural Area of China, 1996-2010. Current Therapeutic Research, 74, 22-25. https://doi.org/10.1016/j.curtheres.2012.12.005

[10] Mbarki, H., Belghiti, K.A., Harmouch, T., Najdi, A. and et Tarik Sqalli, M.A. (2016) Ponction biopsies rénales dans le Service de Néphrologie de Fès: indications et résultats: à propos de 522 cas. Pan African Medical Journal, 24, 39-82. http://www.panafrican-med-journal.com/content/article/24/21/full/ https://doi.org/10.11604/pamj.2016.24.21.3982

[11] (2020) Institut national de la statistique et des études économiques. https://www.insee.fr/fr/statistiques/2381476

[12] Polito, M.G., de Moura, L.A. and Kirsztajn, G.M. (2010) An Overview on Frequency of Renal Biopsy Diagnosis in Brazil: Clinical and Pathological Patterns Based on 9617 Native Kidney Biopsies. Nephrology Dialysis Transplantation, 25, 490-496. https://doi.org/10.1093/ndt/gfp355

[13] Meyrier, A. (2015) Nephrosclerosis: Update on a Centenarian. Nephrology Dialysis Transplantation, 30, 1833-1841. https://doi.org/10.1093/ndt/gfu366

[14] Suganuma, T. (1994) Glomerular IgA Deposits in an Autopsy Study. Nihon Jinzo 
Gakkai Shi, 36, 813-822.

[15] Cattran, D.C., Coppo, R., Cook, H.T., Feehally, J., Robert, I.S.D., Troyanov, S., et al. (2009) The Oxford Classification of IgA Nephropathy: Rationale, Clinicopathological Correlations, and Classification. Kidney International, 76, 534-545. https://doi.org/10.1038/ki.2009.243

[16] Assogba, G.F.A., Couchoud, C., Roudier, C., Pornet, C., Fosse, S., Romon, I., et al. (2012) Prevalence, Screening and Treatment of Chronic Kidney Disease in People with Type 2 Diabetes in France: The Entred Surveys (2001 and 2007). Diabetes \& Metabolism, 38, 558-566. https://doi.org/10.1016/j.diabet.2012.08.004

[17] Wirta, O., Helin, H., Mustonen, J., Kuittinen, E., Savela, T. and et Pasternack, A (2000) Renal Findings and Glomerular Pathology in Diabetic Subjects. Nephron, 84, 236-242. https://doi.org/10.1159/000045583

[18] Choukroun, G., Gupy, Ch. and Noel, Lh. (2000) Amylose du rein et de l'appareil urinaire. In: Grateau, G., Benson, M.D. and Delpech, M., Eds., Les amyloses, Médecine-sciences, Flammarion, Paris, 163-177.

[19] Waldo, B. (2009) The Value of Post-Biopsy Ultrasound in Predicting Complications after Percutaneous Renal Biopsy of Native Kidneys. Nephrology Dialysis Transplantation, 24, 2433-2439. https://doi.org/10.1093/ndt/gfp073

[20] Peters, B., Andersson, Y., Stegmayr, B., Mölne, J., Jensen, G., Dahlberg, P., et al. (2014) A Study of Clinical Complications and Risk Factors in 1001 Native and Transplant Kidney Biopsies in Sweden. Acta Radiologica, 55, 890-896.

https://doi.org/10.1177/0284185113506190 